\title{
Classical Stückelberg interferometry of a nanomechanical two-mode system
}

\author{
Maximilian J. Seitner,,${ }^{1,2, *}$ Hugo Ribeiro, ${ }^{3}$ Johannes Kölbl, ${ }^{1}$ Thomas Faust, ${ }^{2}$ Jörg P. Kotthaus, ${ }^{2}$ and Eva M. Weig ${ }^{1,2}$ \\ ${ }^{1}$ Departement of Physics, University of Konstanz, 78457 Konstanz, Germany \\ ${ }^{2}$ Center for NanoScience (CeNS) and Fakultät für Physik, Ludwig-Maximilians-Universität, \\ Geschwister-Scholl-Platz, 1, München 80539, Germany \\ ${ }^{3}$ Department of Physics, McGill University, Montreal, Quebec, Canada H3A 2 T8
}

(Received 1 July 2016; revised manuscript received 20 October 2016; published 5 December 2016)

\begin{abstract}
Stückelberg interferometry is a phenomenon that has been well established for quantum-mechanical two-level systems. Here, we present classical two-mode interference of a nanomechanical two-mode system, realizing a classical analog of Stückelberg interferometry. Our experiment relies on the coherent energy exchange between two strongly coupled, high-quality factor nanomechanical resonator modes. Furthermore, we discuss an exact theoretical solution for the double-passage Stückelberg problem by expanding the established finite-time LandauZener single-passage solution. For the parameter regime explored in the experiment, we find that the Stückelberg return probability in the classical version of the problem formally coincides with the quantum case, which reveals the analogy of the return probabilities in the quantum-mechanical and the classical version of the problem. This result qualifies classical two-mode systems at large to simulate quantum-mechanical interferometry.
\end{abstract}

DOI: 10.1103/PhysRevB.94.245406

\section{INTRODUCTION}

In 1932, Stückelberg [1] investigated the dynamics of a quantum two-level system undergoing a double passage through an avoided crossing. For a given energy splitting, an interference pattern arises that depends on the transit time and the rate at which the energy of the system is changed. This discovery led to the advent of Stückelberg interferometry, which allows for characterizing the parameters of a two-level system or for achieving quantum control over the system [2]. Stückelberg interferometry has been studied intensively in a variety of quantum systems, e.g., Rydberg atoms [3], ultracold atoms and molecules [4], dopants [5], nanomagnets [6], quantum dots [7-10], and superconducting qubits [11-15], as well as theoretically in a semiclassical optomechanical approach [16]. Here, we study experimentally a classical analog of Stückelberg interferometry, i.e., the coherent energy exchange of two strongly coupled classical high- $Q$ nanomechanical resonator modes. We employ the analytical solution [17] of the Landau-Zener problem describing the single passage through the avoided crossing [1,18-20] to analyze the Stückelberg problem, demonstrating that the classical coherent exchange of energy follows the same dynamics as the coherent tunneling of a quantum-mechanical two-level system.

The past few years have seen the advent of highly versatile nanomechanical systems based on strongly coupled, highquality factor modes [21-23]. The strong coupling generates a pronounced avoided crossing of the classical mechanical modes realizing a nanomechanical two-mode system that can be employed as a testbed for the dynamics at energy level crossings [21-24].

In the case of a quantum two-level system, e.g., spin- $1 / 2$, a single passage through the avoided crossing results in LandauZener dynamics originating from the tunneling of a quantummechanical excitation between two quantum states [18]. In the classical case, the exchange of excitation energy between

*maximilian.seitner@uni-konstanz.de two strongly coupled mechanical modes represents a wellestablished analogy to this process [25,26].

During a double passage through the avoided crossing within the coherence time of the system, phase is accumulated, leading to self-interference. This interference results in oscillations of the return probability, in a quantum-mechanical context well-known as Stückelberg oscillations [1], which have previously been studied in many quantum systems $[7-9,15,27]$. In the classical case, the return probability is analogous to the probability that the excitation, namely oscillation energy, returns coherently to the same mechanical mode.

\section{NANOMECHANICAL TWO-MODE SYSTEM}

We explore experimentally a purely classical mechanical two-mode system, consisting of two orthogonally polarized fundamental flexural modes of a nanomechanical resonator [Fig. 1(a)]. The flexural modes belong to the in-plane and out-of-plane vibration of a 50- $\mu \mathrm{m}$-long, 270 -nm-wide, and 100-nm-thick doubly clamped, high-stress silicon nitride ( $\mathrm{SiN}$ ) string resonator. Dielectric drive and control via electric gradient fields [28] as well as the microwave cavity enhanced heterodyne dielectric detection scheme [22,28,29] are provided via two adjacent gold electrodes, as detailed in Appendix A. Applying a dc voltage to the electrodes induces an electric polarization in the silicon nitride string, which, in turn, couples to the electric field gradient, resulting in a quadratic resonance frequency shift with the applied voltage [28]. The electric field gradients along the in- and out-of-plane direction have opposing signs, and hence they have an inverse tuning behavior. Whereas the out-of-plane oscillation shifts to higher mechanical resonant frequencies, the in-plane oscillation decreases in frequency with the applied dc voltage [28]. Hence, the inherent frequency offset of inplane and out-of-plane oscillation, induced by the rectangular cross section of the string, can be compensated. Furthermore, the applied inhomogeneous electric field induces a strong coupling between the two modes [24]. Near resonance, they hybridize into normal modes [22], diagonally polarized along 
(a)
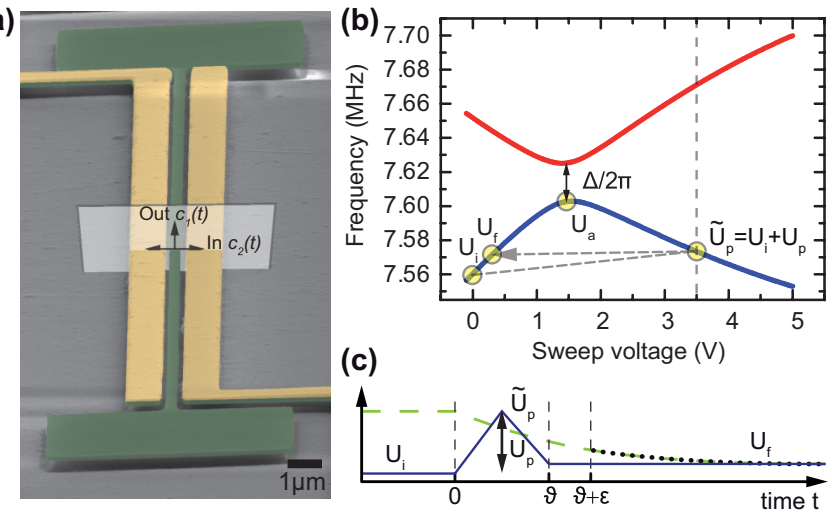

FIG. 1. Nanomechanical resonator and measurement scheme. (a) False color scanning electron micrograph of the 50- $\mu \mathrm{m}$-long, 270-nm-wide, and 100-nm-thick silicon nitride string (green) flanked by two adjacent gold electrodes in the oblique view. Arrows indicate the flexural mode polarizations out-of-plane (Out) and in-plane (In). The normalized amplitude of the respective mode is denoted $c_{1}(t)$ for out-of-plane polarization and $c_{2}(t)$ for in-plane polarization, as explained in the text. (b) Avoided mode crossing of sample A exhibiting a frequency splitting of $\Delta / 2 \pi=22.6 \mathrm{kHz}$ at the avoided crossing voltage $U_{\mathrm{a}}=U_{\mathrm{i}}+1.47 \mathrm{~V}=9.37 \mathrm{~V}$. The lower (out-ofplane) mode is excited at frequency $\omega_{1}\left(U_{\mathrm{i}}\right) / 2 \pi=7.560 \mathrm{MHz}$ defined by the initialization voltage $U_{\mathrm{i}}$. An additional sweep voltage applies a triangular voltage ramp rising to a maximum of $U_{\mathrm{p}}$ and back to the readout voltage $U_{\mathrm{f}}=U_{\mathrm{i}}+0.2 \mathrm{~V}=8.1 \mathrm{~V}$, thus transgressing the avoided crossing twice. The sweep voltage also decouples the mode from the fixed-frequency drive, consequently inducing an exponential decay of the amplitude. (c) Time evolution of the sweep voltage beginning at $t=0$, increasing to $U_{\mathrm{p}}$ and returning to $U_{\mathrm{f}}$ after interval $\vartheta$. The mechanical signal power (green dashed line) is measured after a delay $\varepsilon$, and a fit (black dotted line) is used to extract its magnitude at time $t=\vartheta$. The measured return signal is normalized to the mechanical signal power at $t=0$.

$\pm 45^{\circ}$. A pronounced avoided crossing with level splitting $\Delta / 2 \pi$ reflects the strong mutual coupling of the flexural mechanical modes as depicted in Fig. 1(b).

To study Stückelberg interferometry, we perform a double passage through the avoided crossing using a fast triangular voltage ramp. We initialize the system at voltage $U_{\mathrm{i}}$ in the lower branch of the avoided crossing via a resonant sinusoidal drive tone at the resonance frequency $\omega_{1}\left(U_{\mathrm{i}}\right) / 2 \pi$ of the out-of-plane oscillation [cf. Fig. 1(b)]. As illustrated in Fig. 1(c), at time $t=0$, a fast triangular voltage ramp with voltage sweep rate $\beta$ up to the peak voltage $U_{\mathrm{p}}$, and back to the readout voltage $U_{\mathrm{f}}$, is applied to tune the system through the avoided crossing. Note that the ramp detunes the system from the resonant drive, and the mechanical energy starts to decay exponentially. At $U_{\mathrm{f}}$, we measure the exponential decay of the mechanical oscillation in the lower branch after time $t=\vartheta+\varepsilon$, where $\vartheta$ is the duration of the ramp, i.e., the propagation time, and $\varepsilon$ serves as a temporal offset to avoid transient effects. The signal is extrapolated and evaluated at time $\vartheta$ by an exponential fit and normalized to the signal intensity at the initialization point $(t=0)$, consequently yielding a normalized squared return amplitude. The return signal has to be measured at the readout voltage $U_{\mathrm{f}}$ at $\omega_{1}\left(U_{\mathrm{f}}\right) / 2 \pi$ since the fixed rf drive tone at $\omega_{1}\left(U_{\mathrm{i}}\right) / 2 \pi$ cannot be turned off during the measurement. The presented voltage sequence is analogous to the one employed in Ref. [27], and it differs from the frequently performed periodic driving scheme in Stückelberg interferometry experiments [2].

\section{FINITE-TIME STÜCKELBERG THEORY}

We follow the work of Novotny [30] to derive the classical flow (Hamiltonian flow [31]) describing the dynamics of the system in the vicinity of the avoided crossing. We start with Newton's equation of motion for the displacement,

$$
\begin{aligned}
& m \ddot{u}_{1}(t)=-k_{1} u_{1}(t)-\kappa\left[u_{1}(t)-u_{2}(t)\right], \\
& m \ddot{u}_{2}(t)=-k_{2} u_{2}(t)+\kappa\left[u_{1}(t)-u_{2}(t)\right],
\end{aligned}
$$

with $u_{j}(t)(j=1,2)$ describing, respectively, the out-of-plane $(j=1)$ and in-plane $(j=2)$ displacement of the center of mass of the oscillator, $k_{j}$ is the spring constant of mode $j$, $\kappa$ is the coupling constant between the two modes, and $m$ is the effective mass of the oscillator. We look for solutions of the form $u_{j}(t)=c_{j}(t) \exp \left(i \tilde{\omega}_{1} t\right)$, with $c_{j}(t)$ a normalized amplitude, i.e., $\left|c_{1}(t)\right|^{2}+\left|c_{2}(t)\right|^{2}=1$, and we have defined $\tilde{\omega}_{j}=\sqrt{\left(k_{j}+\kappa\right) / m}$ as the dressed resonance frequency of mode $j$ in units of $2 \pi$. In the experimentally relevant limit where $\kappa / k_{1} \ll 1$, the amplitudes $c_{j}(t)$ are slowly varying in time as compared to the oscillatory function $\exp \left(i \tilde{\omega}_{1} t\right)$. As a consequence, it is possible to neglect the second derivates $\ddot{c}_{j}(t)$ in the equations describing the motion of $c_{j}(t)$, which are obtained by replacing the ansatz for $u_{j}(t)$ in Eq. (1). Thus, the system of coupled differential equations describing the evolution of the normalized amplitudes is

$$
\begin{aligned}
i \dot{c}_{1} & =\frac{\kappa}{2 \tilde{\omega}_{1} m} c_{2}, \\
i \dot{c}_{2} & =\frac{\kappa}{2 \tilde{\omega}_{1} m} c_{1}-\frac{\tilde{\omega}_{2}^{2}-\tilde{\omega}_{1}^{2}}{2 \tilde{\omega}_{1}} c_{2} .
\end{aligned}
$$

In the vicinity of the avoided crossing, where the modes can exchange energy, we have $\tilde{\omega}_{2} \simeq \tilde{\omega}_{1}$ such that $\left(\tilde{\omega}_{2}^{2}-\tilde{\omega}_{1}^{2}\right) / 2 \tilde{\omega}_{1} \simeq$ $\tilde{\omega}_{2}-\tilde{\omega}_{1}$. If we further assume $\tilde{\omega}_{2}-\tilde{\omega}_{1} \simeq \alpha t$, with $\alpha$ the frequency sweep rate, and we define $\Delta=|\lambda|=\kappa /\left(m \tilde{\omega}_{1}\right)$, Eq. (2) reduces to

$$
i \dot{\mathbf{c}}(t)=H(t) \mathbf{c}(t),
$$

with $\mathbf{c}(t)=\left[c_{1}(t) c_{2}(t)\right]^{\mathrm{T}}$ and

$$
H(t)=\left(\begin{array}{cc}
0 & \frac{\lambda}{2} \\
\frac{\lambda}{2} & -\alpha t
\end{array}\right) .
$$

Since we are interested in multiple passages through the avoided crossing, we look for the classical flow $\varphi\left(t, t_{\mathrm{i}}\right)$ defining the state of the system at time $t$ given that we know its state at some prior time $t_{\mathrm{i}}, \mathbf{c}(t)=\varphi\left(t, t_{\mathrm{i}}\right) \mathbf{c}\left(t_{\mathrm{i}}\right)$. Typically, $\mathbf{c}\left(t_{\mathrm{i}}\right)$ is the initial condition of the system. One can show that the classical flow obeys the same differential equation as $\mathbf{c}(t), i \dot{\varphi}\left(t, t_{\mathrm{i}}\right)=H(t) \varphi\left(t, t_{\mathrm{i}}\right)$. By applying the time-dependent unitary transformation $S(t)=\exp \left(i \alpha t^{2} / 4\right) \mathbb{1}_{2}$ to the classical flow, i.e., $\varphi\left(t, t_{\mathrm{i}}\right)=S(t) \tilde{\varphi}\left(t, t_{\mathrm{i}}\right) S^{\dagger}\left(t_{\mathrm{i}}\right)$, we find that $\tilde{\varphi}\left(t, t_{\mathrm{i}}\right)$ obeys the differential equation,

$$
\begin{aligned}
i \dot{\tilde{\varphi}}\left(t, t_{\mathrm{i}}\right) & =\left(S^{\dagger}(t) H(t) S(t)-i S^{\dagger}(t) \dot{S}(t)\right) \tilde{\varphi}\left(t, t_{\mathrm{i}}\right) \\
& =\tilde{H}(t) \tilde{\varphi}\left(t, t_{\mathrm{i}}\right),
\end{aligned}
$$




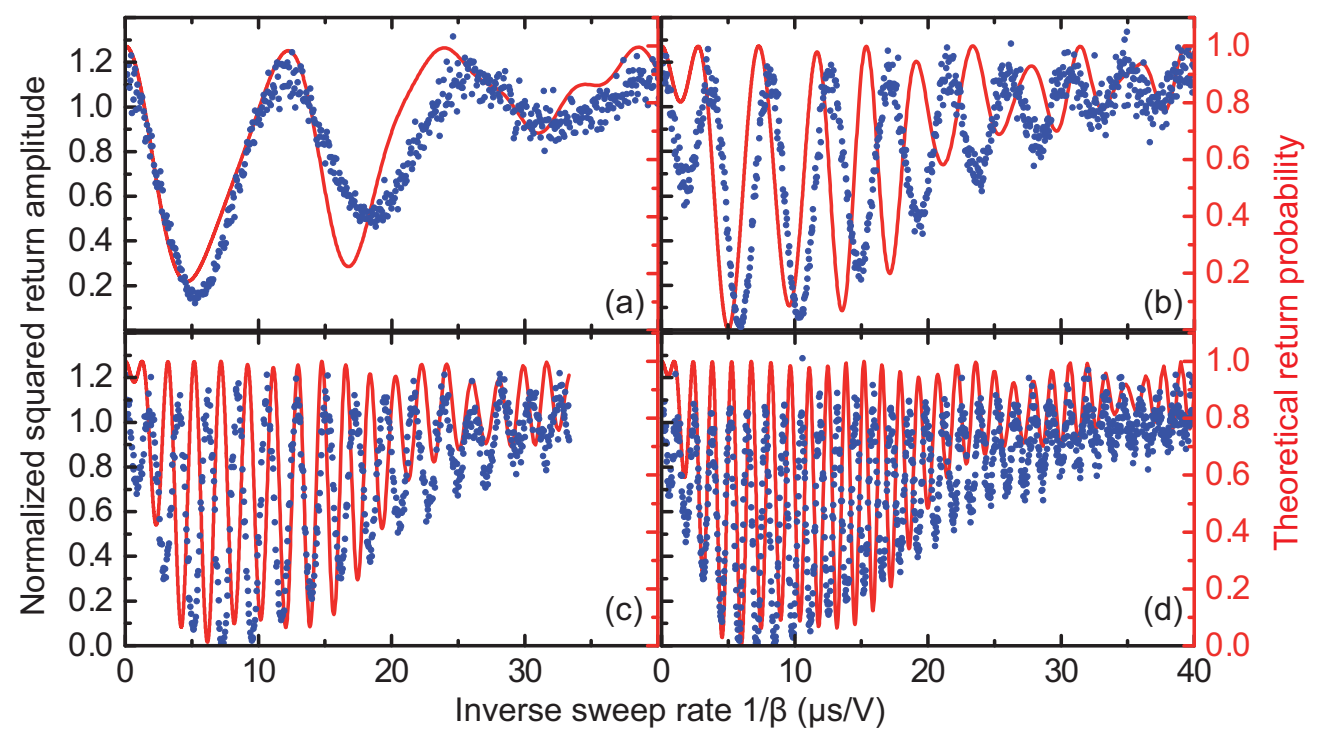

FIG. 2. Classical Stückelberg oscillations. Normalized squared return amplitude (left axis, blue dots) and theoretically calculated return probability (right axis, red line) vs inverse sweep rate for fixed peak voltages of $U_{\mathrm{p}}=2.5 \mathrm{~V}$ (a), $U_{\mathrm{p}}=3.5 \mathrm{~V}$ (b), $U_{\mathrm{p}}=4.5 \mathrm{~V}$ (c), and $U_{\mathrm{p}}=5.0 \mathrm{~V}(\mathrm{~d})$ measured on sample A.

with

$$
\tilde{H}(t)=\left(\begin{array}{cc}
\frac{\alpha t}{2} & \frac{\lambda}{2} \\
\frac{\lambda}{2} & -\frac{\alpha t}{2}
\end{array}\right),
$$

where $\mathbb{1}_{2}$ denotes the unity operator in two dimensions. Equation (5) coincides with the Schrödinger equation for the unitary evolution operator of the Landau-Zener problem, for which an exact finite-time solution is known [17] (see also Appendix B). With the help of the classical flow, one can easily calculate the state of the system after a double passage through the avoided crossing (Stückelberg problem). We find

$$
\mathbf{c}(t)=\varphi_{\mathrm{b}}\left(t,-t_{\mathrm{p}}\right) \varphi\left(t_{\mathrm{p}}, t_{\mathrm{i}}\right) \mathbf{c}\left(t_{\mathrm{i}}\right)
$$

with $\varphi_{\mathrm{b}}\left(t, t_{\mathrm{i}}\right)=\sigma_{x} \varphi\left(t, t_{\mathrm{i}}\right) \sigma_{x}$ describing the evolution of the system during the back sweep (see Appendix B) where $\sigma_{x}$ denotes the Pauli matrix in the $x$ direction, and $t_{\mathrm{p}}$ labels the time at which the forward (backward) sweep stops (starts). From Eq. (7), one can obtain the return probability to mode 1,

$$
P_{1 \rightarrow 1}=\left|\varphi_{11}\left(t_{\mathrm{p}}, t_{\mathrm{i}}\right) \varphi_{11}^{*}\left(t,-t_{\mathrm{p}}\right)+\varphi_{12}^{*}\left(t_{\mathrm{p}}, t_{\mathrm{i}}\right) \varphi_{12}^{*}\left(t,-t_{\mathrm{p}}\right)\right|^{2},
$$

with $\varphi_{i j}\left(t, t_{\mathrm{i}}\right)$ the matrix elements of $\varphi\left(t, t_{\mathrm{i}}\right)$. Note that we use the frequency sweep rate $\alpha$ in the theory, which is converted to the experimentally accessible voltage sweep rate $\beta$ via a conversion factor $\zeta=55 \mathrm{kHz} / \mathrm{V}$ as elucidated in Appendix C.

The analogy between the unitary evolution operator and the classical flow, both expressed in the basis of uncoupled states (modes), allows one to draw the analogy to the quantummechanical return probability in Stückelberg interferometry. The normalized amplitudes are associated with the normalized energy in each resonator mode, and they differ conceptually from the probability that a quantum-mechanical two-level system is found in either of the two quantum states. Nevertheless, the dynamics of the normalized amplitudes in classical Stückelberg interferometry is analogous to the dynamics of the quantum-mechanical probabilities since they follow the same equations. In this sense, the coherent exchange of oscillation energy between two coupled modes can be associated with the transfer of population between two quantum states. A more detailed discussion and comparison of our theoretical approach to previous models $[2,17,25,26]$ reveals previously uncharted parameter regimes in Stückelberg interferometry, and it will be presented elsewhere [32].

\section{CLASSICAL STÜCKELBERG INTERFEROMETRY}

Experimentally, we investigate classical Stückelberg oscillations with two different samples in a vacuum of $\leqslant 10^{-4}$ mbar. Sample $\mathrm{A}$ is investigated at $10 \mathrm{~K}$ in a temperature-stabilized pulse tube cryostat, which offers a greatly enhanced stability of the electromechanical system against temperature fluctuations. Sample B is explored at room temperature in order to confirm the results and to check their stability under ambient temperature fluctuations. Note that in both experiments, the system operates deeply in the classical regime [22], and it does not exhibit any quantum-mechanical properties. Sample A exhibits a mechanical quality factor $Q=$ $\omega / \Gamma \approx 2 \times 10^{5}$ and linewidth $\Gamma / 2 \pi \approx 40 \mathrm{~Hz}$ at resonance frequency $\omega_{1}\left(U_{\mathrm{i}}\right) / 2 \pi=7.560 \mathrm{MHz}$ of the $50-\mu \mathrm{m}$-long string resonator ensuring classical coherence times in the millisecond regime [22]. The level splitting $\Delta / 2 \pi=22.6 \mathrm{kHz}$ exceeds the mechanical linewidth by almost three orders of magnitude, which puts the system deep into the strong-coupling regime.

We initialize the system at $U_{\mathrm{i}}=7.9 \mathrm{~V}$ and apply triangular voltage ramps with different voltage sweep rates $\beta$ for a set of peak voltages $U_{\mathrm{p}}$. Figure 2 depicts the normalized squared return amplitude for different peak voltages and the theoretical return probabilities calculated without any free parameters. The normalized squared return amplitude may exceed a value of unity due to normalization artefacts that arise from the different signal magnitudes at the initialization and readout voltages in addition to measurement errors. We observe clear oscillations in the return signal, in good agreement with the theoretical predictions for lower peak voltages. As the number of oscillations increases for higher peak voltages, the 
(a)

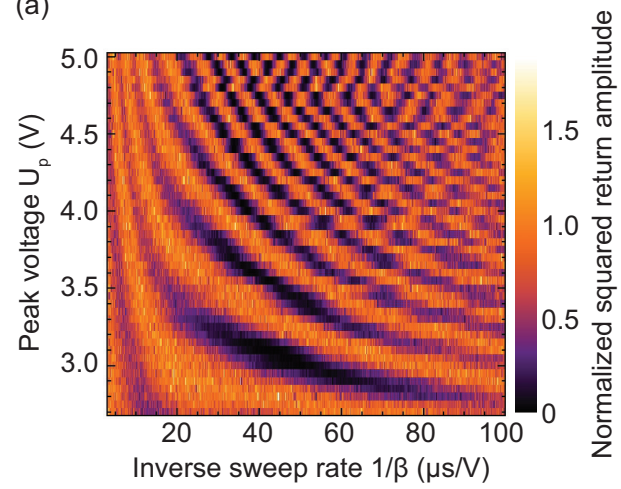

(b)

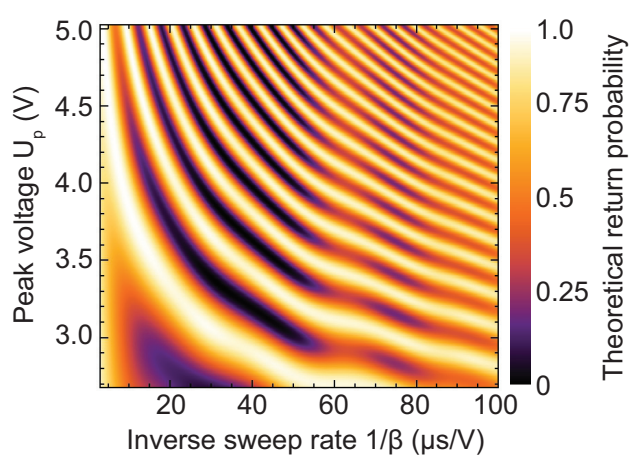

FIG. 3. Comparison of experimental data and the theoretical model. (a) Color-coded normalized squared return amplitude vs inverse sweep rate and peak voltage measured on sample B. The dataset is not interpolated. (b) Color-coded theoretical return probability given by Eq. (9) vs inverse sweep rate and peak voltage for the equivalent data range. The theory is calculated with a single set of parameters, extracted from the avoided crossing illustrated in Appendix C (Fig. 7), and it contains no free parameters.

deviation from the theoretical prediction is more pronounced. We attribute this to uncertainties and fluctuations of the characteristic sweep parameters of the system, which change under application of the voltage ramp and over time as discussed in Appendixes D and E. A further deviation arises from the assumption that a linear change of the voltage leads to a linear change of the difference in frequency. This is only an approximation since the mechanical resonance frequencies tune quadratically with the applied voltage [28]. However, since most of the energy exchange happens in the vicinity of the avoided crossing, where the difference in frequency is linearized, one expects to see noticeable deviations from theory only for higher peak voltages.

To reproduce the experimental data and to test the stability of classical Stückelberg interferometry against fluctuations, we repeat the experiment on a second sample of the same design at room temperature (sample B, denoted by index " $\mathrm{B}$ "). The now 55- $\mu \mathrm{m}$-long resonator has a mechanical linewidth of $\Gamma_{\mathrm{B}} / 2 \pi \approx 25 \mathrm{~Hz}$ at frequency $\omega_{\mathrm{B}, 1}\left(U_{\mathrm{B}, \mathrm{i}}\right) / 2 \pi=6.561 \mathrm{MHz}$, which results in a quality factor of $Q_{\mathrm{B}} \approx 2.6 \times 10^{5}$ at the initialization voltage $U_{\mathrm{B}, \mathrm{i}}=10.4 \mathrm{~V}$ and hence an improved mechanical lifetime of $6.21 \mathrm{~ms}$. Furthermore, the sample exhibits a mode splitting of $\Delta_{\mathrm{B}} / 2 \pi=6.3 \mathrm{kHz}$ and a conversion factor of $\zeta_{\mathrm{B}}=19 \mathrm{kHz} / \mathrm{V}$.

Figure 3 depicts a color-coded two-dimensional map of the normalized squared return amplitude as a function of the inverse voltage sweep rate $\beta$ and the peak voltage $U_{\mathrm{p}}$ alongside the theoretical return probability of the classical Stückelberg oscillations, again calculated with no free parameters. We investigate double passages up to a total propagation time of $\vartheta=$ $1.0 \mathrm{~ms}$ in the experiments conducted on sample B. To account for the decay of both modes when tuned away from the drive for the considerably longer ramps applied to sample B, we model the mechanical damping by an exponential decay with an averaged decay time $t_{0}=5.7 \mathrm{~ms}$. After a measurement time $t_{m}$, the probability to measure an excitation of mode $j$ is given by

$$
\left|c_{j}\left(t_{m}\right)\right|^{2}=\exp \left(-t_{m} / t_{0}\right) P_{1 \rightarrow j}
$$

with $P_{1 \rightarrow 1}$ given by Eq. (8) and $P_{1 \rightarrow 2}=1-P_{1 \rightarrow 1}$. The experimental data show remarkably good agreement with the theoretical predictions, despite temperature fluctuations of several degrees kelvin per hour, which shift the mechanical resonance frequency up to 40 linewidths. To initialize the system at the same resonance frequency in each measurement, a feedback loop regulates the initialization voltage $U_{\mathrm{i}}$ (see Appendix D). Consequently, the recording of a single horizontal scan at a fixed peak voltage in Fig. 3(a) takes up to 16 hours, incorporating a non-negligible amount of fluctuations of the system parameters, such as, e.g., the center voltage of the avoided crossing $U_{\mathrm{a}}$, which imposes considerable uncertainties on the parameters used for the theoretical calculations. To further illustrate the influence of fluctuations, Figs. 4(a) and 4(b) depict horizontal and vertical

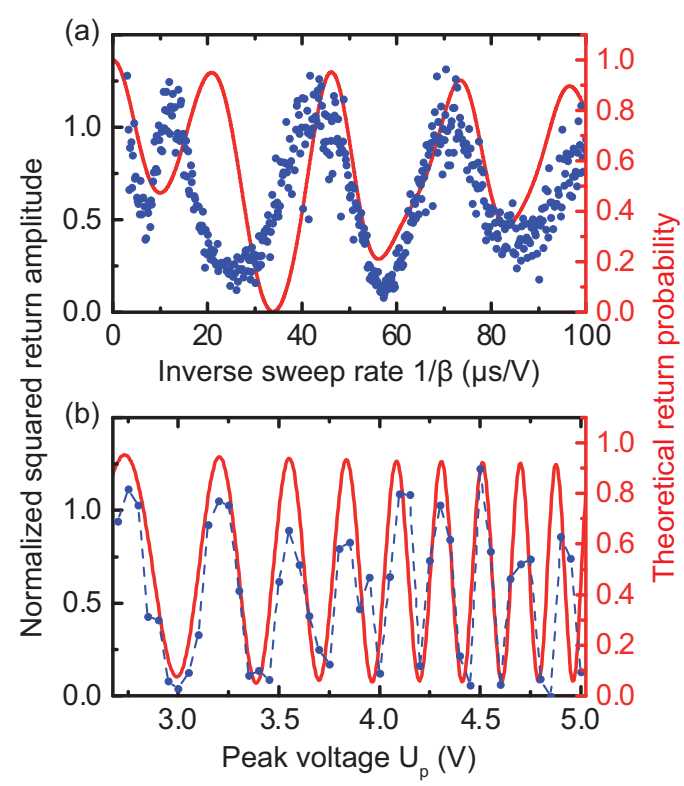

FIG. 4. Exemplary classical Stückelberg oscillations of sample B. Line cuts of Figs. 3(a) and 3(b). (a) Normalized squared return amplitude (left axis, blue dots) and theoretically calculated return probability given by Eq. (9) (right axis, red line) vs inverse sweep rate for a fixed peak voltage $U_{\mathrm{p}}=3.3 \mathrm{~V}$. (b) The same quantities as above but plotted as a function of peak voltage for a fixed inverse sweep rate $1 / \beta=51.6 \mu \mathrm{s} / \mathrm{V}$. Blue dots are joined by blue dashed lines for illustration reasons. 
line cuts of the two-dimensional map in Fig. 3 at $U_{\mathrm{p}}=3.3 \mathrm{~V}$ and at inverse sweep rate $1 / \beta=51.6 \mu \mathrm{s} / \mathrm{V}$, respectively. For small inverse sweep rates, i.e., very fast sweeps, the experimental data in Fig. 4(a) deviate from the theoretical model due to a flattening of the voltage ramps in the roomtemperature experiment (see Appendix E). For sweeps with $1 / \beta \geqslant 50 \mu \mathrm{s} / \mathrm{V}$, the experimentally observed Stückelberg oscillations exhibit good agreement with the theoretical predictions even for the line cut along the vertical peak voltage axis [cf. Fig. 4(b)]. Note that Fig. 4 depicts the best results from all datasets at room temperature. Further exemplary line cuts are provided in Appendix E, also exhibiting a clear oscillatory behavior in the normalized squared return amplitude, but incorporating larger deviations from theory in certain regions and therefore revealing fluctuations of system parameters over time, predominately induced by temperature drifts.

\section{CONCLUSION AND OUTLOOK}

In conclusion, we have demonstrated classical Stückelberg oscillations that have previously been experimentally observed exclusively in the framework of quantum mechanics $[2,7,8,27]$. An exact solution of the Stückelberg problem [1] based on the finite-time Landau-Zener single-passage solution [17] describes the return probability in the classical version of the double-passage problem, which is shown to follow the same equation as in the case of a quantum twolevel system. In this way, we have demonstrated that in our experimental parameter limit, the coherent exchange of energy between two strongly coupled classical nanomechanical resonator modes follows the same dynamics as the exchange of excitations in a quantum-mechanical two-level system in the framework of Stückelberg interferometry.

This analogy might be exploited in future experiments in order to determine whether a system of coupled mechanical modes operates in the classical regime or in the quantum-mechanical limit. In the general case of two coupled quantum harmonic oscillators, the effective model describing the dynamics would resemble that of the multiple-crossing Landau-Zener problem [33], which leads to a much more complex dynamics than the provided solution for the classical case. Hence, if one would be able to design an experiment in which two strongly coupled mechanical oscillators can be cooled to their quantum-mechanical limit, it should be possible to determine if the system operates in the classical or the quantum regime by means of a simple Stückelberg return amplitude measurement rather than by probing the Wigner distribution function [34]. Whereas the presented experimental setup is still far from operation in the quantum-mechanical limit, we could imagine this technique to be applied to different mechanical systems that already demonstrated the quantum limit of mechanical oscillators [35,36].

Overall, we have found good agreement between experiment and theory. However, parameter regimes yielding larger deviations are reminiscent of the sensitivity of the exact Stückelberg solution to the initial system parameters, such as the position of the avoided crossing, and hence to fluctuations in the system. This circumstance, in turn, might be exploited for future investigations in resonator metrology of decoherence and noise, adapting the approach to employ
Stückelberg interferometry to characterize the coherence of a qubit [10].

Furthermore, the possibility to create a superposition state of two mechanical modes may allow for future application as highly sensitive nanomechanical interferometers [37-39] analogous to the applications with cold atom and molecule matter-wave interferometers [4,40-42], whereas the presence and implications of, e.g., phase noise [43] can be resolved by a change in resonator population and interference pattern.

Finally, classical Stückelberg interferometry should not be limited to the presented strongly coupled, high-quality factor nanomechanical string resonator modes [22], but it can be observed in principle in every classical two-mode system exhibiting the possibility of a double passage through an avoided crossing within the classical coherence time.

\section{ACKNOWLEDGMENTS}

Financial support by the Deutsche Forschungsgemeinschaft via the collaborative research center SFB 767 and via project Ko 416-18 is gratefully acknowledged. H. R. acknowledges funding from the Swiss SNF. We thank Aashish A. Clerk for a critical reading of the manuscript.

\section{APPENDIX A: THE NANOELECTROMECHANICAL SYSTEM}

The nanomechanical device and experimental setup are depicted in Fig. 5. The sample investigated at a temperature of $10 \mathrm{~K}$ (sample A) consists of a 50- $\mu \mathrm{m}$-long, 270-nmwide, and 100-nm-thick doubly clamped silicon nitride (SiN) string resonator. The room-temperature measurements were (a)

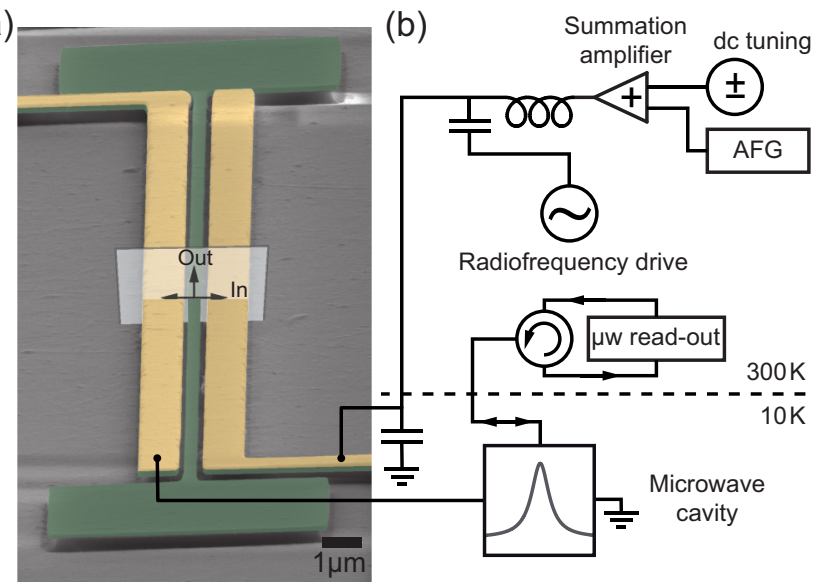

FIG. 5. Nanoelectromechanical system. (a) False color scanning electron micrograph of the 50- $\mu \mathrm{m}$-long, 270-nm-wide, and 100-nmthick silicon nitride string (green) in the oblique view. The adjacent $1-\mu \mathrm{m}$-wide gold electrodes (yellow) are processed on top of the silicon nitride layer. Arrows indicate the flexural mode polarizations out-of-plane (Out) and in-plane (In). (b) Electrical transduction setup. The arbitrary function generator (AFG) ramp voltage and the $\mathrm{dc}$ tuning voltage are added via a summation amplifier and then combined with the rf drive using a bias tee. The microwave readout is bypassed by the second capacitor, acting as a ground path for the microwave cavity. 


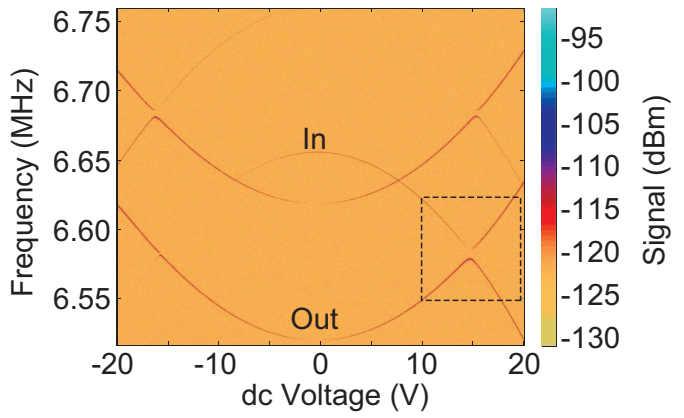

FIG. 6. Dielectric frequency tuning. Color-coded frequency spectrum of sample B as a function of applied dc tuning voltage. The resonance frequency of the 55- $\mu \mathrm{m}$-long resonator's fundamental out-of-plane oscillation (Out) increases quadratically as a function of dc voltage. The resonance frequency of the corresponding in-plane mode (In) decreases quadratically. Tuning both modes into resonance, they exhibit a pronounced avoided crossing indicated by the black dashed rectangle. This particular region is displayed in Fig. 7(a). The additional resonances in the spectrum originate from a different mechanical resonator, which is coupled to the same microwave cavity.

conducted on a similar sample (sample B), differing only in its resonator length of $55 \mu \mathrm{m}$. As stated in the main text, the temperature does not affect the purely classical character of the system. The string resonators exhibit a high intrinsic tensile pre-stress of $\sigma_{\mathrm{SiN}}=1.46 \mathrm{GPa}$ resulting from the LPCVD deposition of the SiN film on the fused silica substrate. This high stress translates into large intrinsic mechanical quality factors of up to $Q \approx 500000$, which reduce quadratically with the applied dc tuning voltage in the experiment as a result of dielectric damping [28]. Dielectric drive, detection, and control are provided via two adjacent gold electrodes in an all integrated microwave cavity enhanced transduction scheme $[22,24,28,29]$. In the experiment, we consider the two orthogonally polarized fundamental flexural modes of the nanomechanical string resonator, namely the oscillation perpendicular to the sample plane (out-of-plane) and the oscillation parallel to the sample plane (in-plane). Applying a dc voltage to one of the two gold electrodes induces an electric polarization in the silicon nitride string resonator, which couples to the field gradient of the inhomogeneous electric field. Consequently, the mechanical resonance frequencies tune quadratically with the applied dc voltage as depicted in Fig. 6. Whereas the out-of-plane resonance (Out) tunes toward higher resonance frequencies as a function of $\mathrm{dc}$ voltage, the resonance frequency of the in-plane mode (In) decreases [28]. Dielectric tuning of both modes into resonance reveals a pronounced avoided crossing originating from the strong mutual coupling induced by the inhomogeneous electric field. In the coupling region, the mechanical modes hybridize into diagonally $\left( \pm 45^{\circ}\right)$ polarized eigenmodes of the strongly coupled system.

\section{APPENDIX B: THEORETICAL MODEL}

In this Appendix, we derive an exact expression for the classical return probability. A detailed discussion and comparison of our theoretical approach to previous models will be published elsewhere [32].

We start by solving the system of first-order differential equations defined in Eq. (5) of the main text. Since these equations are formally identical to the Schrödinger equation for the (quantum) Landau-Zener problem, we can follow the work of Vitanov et al. [17] to derive the classical flow, $\tilde{\mathbf{c}}(\tau)=\tilde{\varphi}\left(\tau, \tau_{\mathrm{i}}\right) \tilde{\mathbf{c}}\left(\tau_{\mathrm{i}}\right)$ with $\tilde{\mathbf{c}}(\tau)=\left[\tilde{c}_{1}(\tau) \tilde{c}_{2}(\tau)\right]^{\mathrm{T}}$. Here, $\tau=\sqrt{\alpha} t$ is a dimensionless time and $\tau_{\mathrm{i}}$ is the initial dimensionless time. Note that we use dimensionless times in this appendix in order to provide a derivation that is consistent with the work of Vitanov et al. [17]. The equations in dependence of times in the main text can be recovered by replacement of the dimensionless times following the above definition. In Appendix C, we provide the explicit conversion from experimentally accessible parameters to the dimensionless times. We find

$$
\left(\begin{array}{c}
\tilde{c}_{1}(\tau) \\
\tilde{c}_{2}(\tau)
\end{array}\right)=\left(\begin{array}{cc}
\tilde{\varphi}_{11}\left(\tau, \tau_{\mathrm{i}}\right) & \tilde{\varphi}_{12}\left(\tau, \tau_{\mathrm{i}}\right) \\
-\tilde{\varphi}_{12}^{*}\left(\tau, \tau_{\mathrm{i}}\right) & \tilde{\varphi}_{11}^{*}\left(\tau, \tau_{\mathrm{i}}\right)
\end{array}\right)\left(\begin{array}{c}
\tilde{c}_{1}\left(\tau_{\mathrm{i}}\right) \\
\tilde{c}_{2}\left(\tau_{\mathrm{i}}\right)
\end{array}\right)
$$

with

$$
\begin{aligned}
& \tilde{\varphi}_{11}\left(\tau, \tau_{\mathrm{i}}\right)=\frac{\Gamma\left(1+i \frac{\eta^{2}}{4}\right)}{\sqrt{2 \pi}}\left[D_{-1-i \frac{\eta^{\frac{2}{4}}}{}}\left(e^{-i \frac{3 \pi}{4}} \tau_{\mathrm{i}}\right) D_{-i \frac{\eta^{2}}{4}}\left(e^{i \frac{\pi}{4}} \tau\right)\right. \\
& \left.+D_{-1-i \frac{\eta^{2}}{4}}\left(e^{i \frac{\pi}{4}} \tau_{\mathrm{i}}\right) D_{-i \frac{\eta^{2}}{4}}\left(e^{-i \frac{3 \pi}{4}} \tau\right)\right]
\end{aligned}
$$

and

$$
\begin{aligned}
\tilde{\varphi}_{12}\left(\tau, \tau_{\mathrm{i}}\right)= & \frac{\Gamma\left(1+i \frac{\eta^{2}}{4}\right)}{\sqrt{2 \pi}} \frac{2}{\eta} e^{-i \frac{\pi}{4}}\left[D_{-i \frac{\eta^{2}}{4}}\left(e^{-i \frac{3 \pi}{4}} \tau_{\mathrm{i}}\right) D_{-i \frac{\eta^{2}}{4}}\left(e^{i \frac{\pi}{4}} \tau\right)\right. \\
& \left.-D_{-i \frac{\eta^{2}}{4}}\left(e^{i \frac{\pi}{4}} \tau_{\mathrm{i}}\right) D_{-i \frac{\eta^{2}}{4}}\left(e^{-i \frac{3 \pi}{4}} \tau\right)\right] .
\end{aligned}
$$

Here, $\eta=\lambda / \sqrt{\alpha}$ is the dimensionless coupling, $\Gamma(z)$ is the Gamma function, and $D_{v}(z)$ is the parabolic cylinder function. To find the flow describing the evolution of the amplitudes defined in Eq. (2), we apply the unitary transformation defined in the main text, $\varphi\left(\tau, \tau_{\mathrm{i}}\right)=S(\tau) \tilde{\varphi}\left(\tau, \tau_{\mathrm{i}}\right) S^{\dagger}\left(\tau_{\mathrm{i}}\right)$, with

$$
S(\tau)=\exp \left[\frac{i}{4} \tau^{2}\right] \mathbb{1}_{2}
$$

We find

$$
\varphi\left(\tau, \tau_{\mathrm{i}}\right)=\exp \left[\frac{i}{4}\left(\tau^{2}-\tau_{\mathrm{i}}^{2}\right)\right] \tilde{\varphi}\left(\tau, \tau_{\mathrm{i}}\right) .
$$

The flow $\varphi\left(\tau, \tau_{\mathrm{i}}\right)$ describes the evolution of the normalized amplitudes for a forward sweep; the frequency of mode 1 (2) increases (decreases) with time. This implies that the back sweep cannot be described by $\varphi\left(\tau, \tau_{\mathrm{i}}\right)$ since during the evolution the frequency of mode 1 (2) decreases (increases). Hence, the system of coupled differential equations describing the dynamics during the backward sweep (denoted by index "b") is given by

$$
i\left(\begin{array}{c}
\dot{\tilde{c}}_{1, \mathrm{~b}} \\
\dot{\tilde{c}}_{2, \mathrm{~b}}
\end{array}\right)=\left(\begin{array}{cc}
-\frac{\alpha t}{2} & \frac{\lambda}{2} \\
\frac{\lambda}{2} & \frac{\alpha t}{2}
\end{array}\right)\left(\begin{array}{l}
\tilde{c}_{1, \mathrm{~b}} \\
\tilde{c}_{2, \mathrm{~b}}
\end{array}\right) .
$$

The solutions of Eq. (B6) can be obtained analogously to the forward flow since the matrices appearing in Eq. (6) of the 
(a)

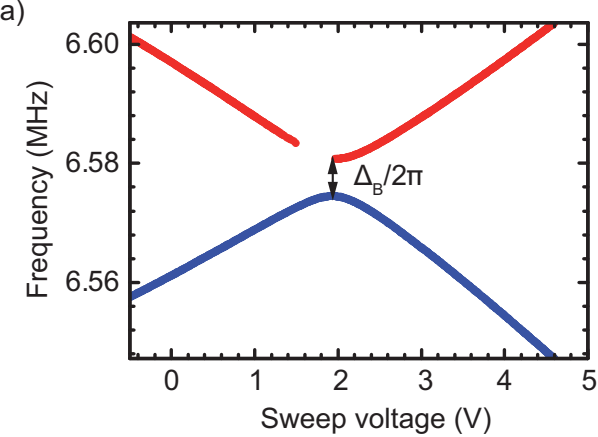

(b)

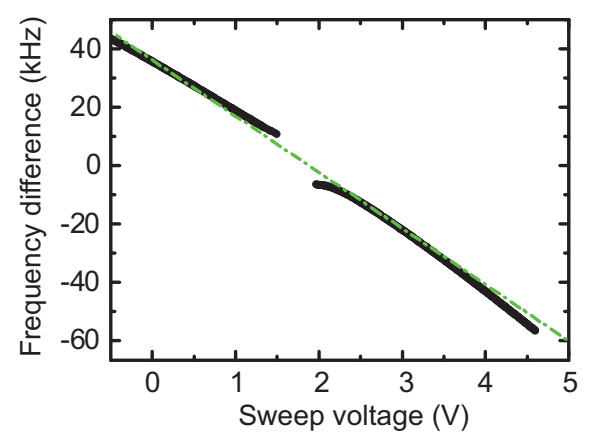

FIG. 7. Calibration of the conversion factor. (a) Avoided crossing region of sample B. A sweep voltage equal to zero corresponds to the initialization point $U_{\mathrm{i}}=10.4 \mathrm{~V}$. The two modes exhibit a frequency splitting of $\Delta_{\mathrm{B}} / 2 \pi=6.3 \mathrm{kHz}$ at the avoided crossing voltage $U_{\mathrm{a}}=U_{\mathrm{i}}+1.96 \mathrm{~V}=12.36 \mathrm{~V}$. The gap in the upper branch (red) results from a signal detection efficiency of the particular mode polarization below the noise level. (b) Frequency difference of the two modes (black) and averaged slope of the linearized frequency tuning illustrated by a green dash-dotted line.

main text and Eq. (B6) are related by a unitary transformation. We find

$$
\tilde{\varphi}_{\mathrm{b}}\left(\tau, \tau_{\mathrm{i}}\right)=\sigma_{x} \tilde{\varphi}\left(\tau, \tau_{\mathrm{i}}\right) \sigma_{x}=\left(\begin{array}{cc}
\tilde{\varphi}_{11}^{*}\left(\tau, \tau_{\mathrm{i}}\right) & -\tilde{\varphi}_{12}^{*}\left(\tau, \tau_{\mathrm{i}}\right) \\
\tilde{\varphi}_{12}\left(\tau, \tau_{\mathrm{i}}\right) & \tilde{\varphi}_{11}\left(\tau, \tau_{\mathrm{i}}\right)
\end{array}\right),
$$

where $\sigma_{x}$ denotes the Pauli matrix in the $x$ direction. The flow describing the evolution of the amplitudes $c_{1}(t)$ and $c_{2}(t)$ during the back sweep is obtained as previously, and we have

$$
\varphi_{\mathrm{b}}\left(\tau, \tau_{\mathrm{i}}\right)=\exp \left[\frac{i}{4}\left(\tau^{2}-\tau_{\mathrm{i}}^{2}\right)\right] \tilde{\varphi}_{\mathrm{b}}\left(\tau, \tau_{\mathrm{i}}\right) .
$$

The state of the system after a double sweep is given by

$$
\mathbf{c}(\tau)=\varphi_{\mathrm{b}}\left(\tau,-\tau_{\mathrm{p}}\right) \varphi\left(\tau_{\mathrm{p}}, \tau_{\mathrm{i}}\right) \mathbf{c}\left(\tau_{\mathrm{i}}\right),
$$

where $\tau_{\mathrm{p}}$ labels the time at which the first sweep stops and $-\tau_{\mathrm{p}}$ corresponds to the initial time of the back sweep [cf. Eq. (7) of the main text]. As stated in Eq. (8) of the main text, the probability to return to mode 1 is then given by

$$
\begin{aligned}
& P_{1 \rightarrow 1}\left(\tau, \tau_{\mathrm{p}}, \tau_{\mathrm{i}}\right) \\
& \quad=\left|\varphi_{11}\left(\tau_{\mathrm{p}}, \tau_{\mathrm{i}}\right) \varphi_{11}^{*}\left(\tau,-\tau_{\mathrm{p}}\right)+\varphi_{12}^{*}\left(\tau_{\mathrm{p}}, \tau_{\mathrm{i}}\right) \varphi_{12}^{*}\left(\tau,-\tau_{\mathrm{p}}\right)\right|^{2} .
\end{aligned}
$$

\section{APPENDIX C: CONVERSION FACTOR CALIBRATION}

In the theoretical model, the state of the system after a double passage through the avoided crossing depends on the characteristic sweep times. Experimentally, we realize this double passage by the application of fast triangular voltage ramps, tuning the resonant frequency of the mechanical modes [28]. In the following, we focus on sample B to illustrate how the different times are obtained.

We initialize the resonance in the lower-frequency branch at the voltage $U_{\mathrm{i}}=10.4 \mathrm{~V}$, where we apply a continuous sinusoidal drive tone at $\omega_{1}\left(U_{\mathrm{i}}\right) / 2 \pi=6.561 \mathrm{MHz}$. We then ramp the sweep voltage up to the peak voltage $U_{\mathrm{p}}$ across the avoided crossing at voltage $U_{\mathrm{a}}=U_{\mathrm{i}}+1.96 \mathrm{~V}=12.36 \mathrm{~V}$ and then back to the readout voltage $U_{\mathrm{f}}=U_{\mathrm{i}}+0.5 \mathrm{~V}=10.9 \mathrm{~V}$, where the oscillation energy is read out again in the lower-frequency branch. The offset of the readout voltage with respect to the initialization voltage is necessary since we cannot stop the sinusoidal drive tone at $\omega_{1}\left(U_{\mathrm{i}}\right) / 2 \pi$ during the experiment. For a fixed peak voltage $U_{\mathrm{p}}$, the voltage sweep is performed for different voltage sweep rates $\beta$, given in the experimental units $[\beta]=\mathrm{V} / \mathrm{s}$. In the theoretical model, the frequency difference of the two modes in units of $2 \pi$ is approximated by $\omega_{2}-\omega_{1} \simeq \alpha t$, where the sweep rate $\alpha$ has the dimensions $[\alpha]=2 \pi \times \mathrm{Hz} / \mathrm{s}$. Consequently, we introduce the conversion factor $\zeta$ from voltage to frequency, defined via the relation

$$
\alpha=2 \pi \times \zeta \beta .
$$

Figure 7 illustrates the calibration of the conversion factor. As is conventional in experiments on Stückelberg interferometry, the frequency difference of the two mechanical modes is approximated to be linear in time, i.e., linear in sweep voltage. In our particular system, the resonance frequencies of the mechanical flexural modes tune quadratically with voltage outside of the avoided crossing (see Fig. 6). Nevertheless, for the designated region around the avoided crossing, the two frequency branches can be linearized as follows. We take the frequency difference of both modes before and after the avoided crossing [cf. Fig. 7(b)], respectively, and we extract the slopes via a linear fit. The two different slopes on the left and the right-hand side of the avoided crossing are averaged, yielding an effective conversion factor (dash-dotted green line)

$$
\zeta=19 \frac{\mathrm{kHz}}{\mathrm{V}}
$$

To estimate the error of the conversion factor, we apply a quadratic fit to the frequency difference of the two modes, and we find a fit residual of approximately $2 \mathrm{kHz} / \mathrm{V}$ between the linear and the quadratic fit.

Depending on the specific peak voltage $U_{\mathrm{p}}$, one could take into account a weighted average of the two slopes in order to mitigate the deviation of the quadratic frequency tuning from the linear approximation. Here, one has to point out deliberately that we neglect any weighted average, but we take solely the above conversion factor for the calculation of the theoretical return probabilities. We are well aware of the fact that this linearization translates into a direct discrepancy between the theoretical model and the experimental results. Nevertheless, in our opinion, these discrepancies are prevailed by the benefits of a closed theoretical calculation using a single set of parameters that is supported by the remarkably good 
agreement between experiment and theory. Hence, we express the characteristic sweep times in the theoretical model by the following parameters extracted from the avoided crossing in Fig. 7(a):

$$
\begin{aligned}
& t_{\mathrm{i}}=-\frac{1}{\beta}\left(U_{\mathrm{a}}-U_{\mathrm{i}}\right)=\frac{\tau_{\mathrm{i}}}{\sqrt{\alpha}}, \\
& t_{\mathrm{p}}=\frac{1}{\beta}\left(\widetilde{U}_{\mathrm{p}}-U_{\mathrm{a}}\right)=\frac{\tau_{\mathrm{p}}}{\sqrt{\alpha}}, \\
& t_{\mathrm{f}}=\frac{1}{\beta}\left(U_{\mathrm{a}}-U_{\mathrm{f}}\right)=\frac{\tau_{\mathrm{f}}}{\sqrt{\alpha}},
\end{aligned}
$$

where $\widetilde{U}_{\mathrm{p}}=U_{\mathrm{i}}+U_{\mathrm{p}}$. As explained above, the return probability is measured at the readout voltage $U_{\mathrm{f}} \neq U_{\mathrm{i}}$. Consequently, we replace $\tau$ by $\tau_{\mathrm{f}}$ in the back sweep of the theory, which modifies Eq. (B10) to

$$
\begin{aligned}
& P_{1 \rightarrow 1}\left(\tau_{\mathrm{f}}, \tau_{\mathrm{p}}, \tau_{\mathrm{i}}\right) \\
& \quad=\left|\varphi_{11}\left(\tau_{\mathrm{p}}, \tau_{\mathrm{i}}\right) \varphi_{11}^{*}\left(\tau_{\mathrm{f}},-\tau_{\mathrm{p}}\right)+\varphi_{12}^{*}\left(\tau_{\mathrm{p}}, \tau_{\mathrm{i}}\right) \varphi_{12}^{*}\left(\tau_{\mathrm{f}},-\tau_{\mathrm{p}}\right)\right|^{2} .
\end{aligned}
$$

\section{APPENDIX D: TEMPERATURE FLUCTUATIONS}

As stated in the main text, the measurement of the normalized squared return amplitude for various voltage sweep rates $\beta$ at a particular peak voltage $U_{\mathrm{p}}$ takes up to $16 \mathrm{~h}$. During this time, the ambient temperature undergoes fluctuations of $\pm 2 \mathrm{~K}$ per hour due to insufficient air conditioning. Since the mechanical resonance frequency shifts due to thermal expansion of the silicon nitride by approximately $500 \mathrm{~Hz} / \mathrm{K}$, both resonances shift by approximately 40 linewidths. To initialize the system at the same resonance frequency for every particular measurement, we implement a feedback loop that regulates the initialization voltage. Therefore, the initialization voltage shifts slightly from measurement to measurement, reflecting the temperature fluctuations. Figure 8 depicts the

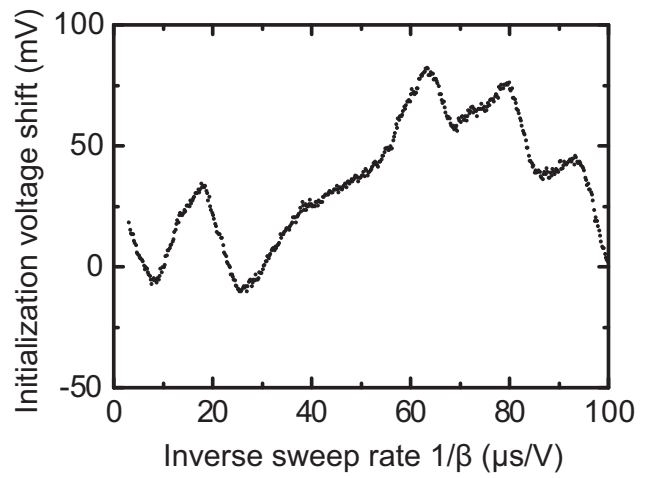

FIG. 8. Temperature fluctuations. Initialization voltage shift vs inverse sweep rate for the dataset depicted in Fig. 4(a) $\left(U_{\mathrm{p}}=3.3 \mathrm{~V}\right)$ of the main text. Each point corresponds to the measurement of the normalized squared return amplitude for a given inverse sweep rate. The first measurement is performed at $1 / \beta=100 \mu \mathrm{s} / \mathrm{V}$, representing the initialization at resonance frequency $\omega_{\mathrm{i}}\left(U_{\mathrm{i}}\right) / 2 \pi$ for voltage $U_{\mathrm{i}}=10.4 \mathrm{~V}$. The implemented feedback loop regulates the initialization voltage in order to compensate for the temperature fluctuations of the mechanical resonance. Consequently, the voltage shift illustrates the fluctuations of the ambient temperature. initialization voltage shift versus inverse sweep rate for the dataset of peak voltage $U_{\mathrm{p}}=3.3 \mathrm{~V}$, which corresponds to the measurement depicted in Fig. 4(a) of the main text. Each point represents a single measurement for a particular sweep rate. The first measurement is performed at an inverse sweep rate of $100 \mu \mathrm{s} / \mathrm{V}$ at the initialization voltage $U_{\mathrm{i}}=10.4 \mathrm{~V}$ and therefore corresponds to a shift of zero volts. Clearly, the temperature fluctuations not only affect the initialization voltage required to obtain the desired resonance frequency, but they will also alter other system parameters, such as the position of the avoided crossing $U_{\mathrm{a}}$, that greatly affect the theory (cf. Appendix C). Consequently, the temperature fluctuations lead to deviations between experiment and theory, since we calculate the return probability with a single set of parameters. In turn, these deviations might be used to infer fluctuations of the system in future applications of Stückelberg interferometry.

\section{APPENDIX E: EXPERIMENTAL UNCERTAINTIES}

In Fig. 9 we provide additional horizontal and vertical line cuts from Fig. 3 of the main text. We observe pronounced oscillations in the normalized squared return amplitude (blue dots) as well as in the theoretically calculated return probability (red line). Nevertheless, the deviations between experiment and theory are more apparent, especially for Fig. 9(b), which depicts a vertical line cut for a fixed inverse sweep rate of $1 / \beta=60 \mu \mathrm{s} / \mathrm{V}$, i.e., within the "plateau" in Fig. 3(b) of the main text. Whereas the normalized squared return amplitude exhibits destructive interference, with the signal dropping

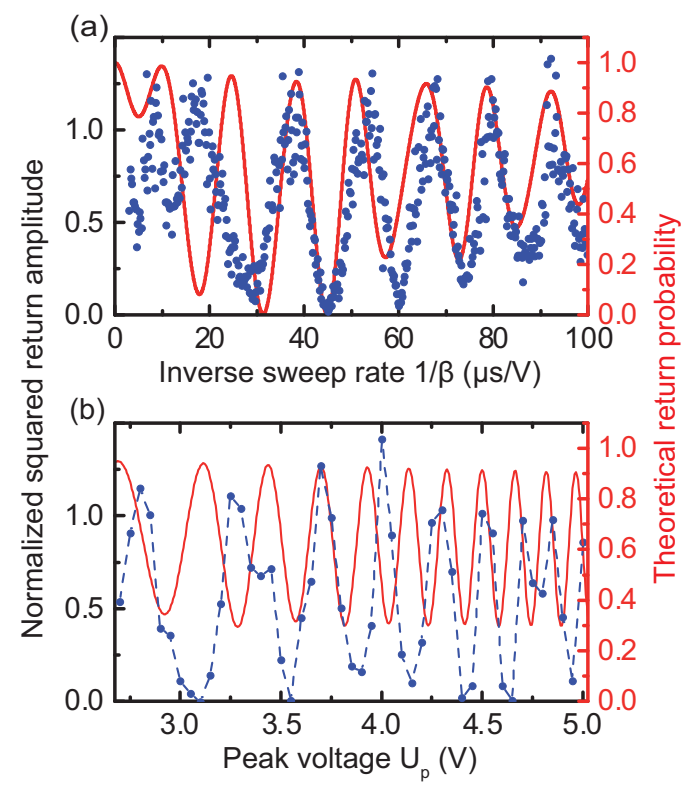

FIG. 9. Classical Stückelberg oscillations. (a) Normalized squared return amplitude (left axis, blue dots) and theoretically calculated return probability given by Eq. (9) of the main text (right axis, red line) vs inverse sweep rate for a fixed peak voltage $U_{\mathrm{p}}=3.85 \mathrm{~V}$. (b) Same quantities as above but plotted as a function of peak voltage for a fixed inverse sweep rate $1 / \beta=60 \mu \mathrm{s} / \mathrm{V}$. Blue dots are joined by blue dashed lines for illustration reasons. 
close to zero, the minima in the return probability saturate at a value of approximately 0.3 . This discrepancy is supposed to originate from the high sensitivity of the theoretical model to the input parameters. Experimental uncertainties and fluctuations deter the system from interference with the same constant parameters throughout all individual measurements. Since the "plateau" in the theory is characteristic for a particular set of exact and constant parameters, it cannot be recovered under the given experimental conditions.

The experimental uncertainties arise not solely from the temperature fluctuations. The voltage ramp also affects the characteristic parameters, such as the exact position of the avoided crossing $U_{\mathrm{a}}$. As previously stated, the dc voltage induces dipoles in the silicon nitride string resonator, which couple to the electric field gradient. A variation in dc voltage changes the inhomogeneous electric field at the same time, to which the nanoelectromechanical system needs to equilibrate. Consequently, the resonance frequencies of the mechanical modes drift toward the equilibrium position of the system. This drift, in turn, alters the characteristic system parameters, i.e., the characteristic voltages used for the theoretical calculations, and it depends on the magnitude of the peak voltage $U_{\mathrm{p}}$. Concerning the initialization voltage, we simultaneously account for this effect via the initialization feedback loop (see Sec. IV). Nevertheless, the exact position of the avoided crossing $U_{\mathrm{a}}$ varies slightly due to this retardation effect. Experimentally, we mitigate the influence of this drift by means of a "thermalization" break of $10 \mathrm{~s}$ after each voltage ramp.
Another possible uncertainty arises from the imprecision in the value of the peak voltage $U_{\mathrm{p}}$ at the sample. The output amplitude uncertainty of the arbitrary function generator used in the room-temperature experiments is classified by the manufacturer as $\pm 1 \%$ of the nominal output voltage. Consequently, the maximum uncertainty in the peak voltage corresponds to $\pm 0.05 \mathrm{~V}$ for a maximum peak voltage of $U_{\mathrm{p}}=5.0 \mathrm{~V}$, which is equal to the voltage step size between two horizontal lines of Fig. 3(a) in the main text.

As stated in the main text, we observed additional deviations in the experimental data of sample B from the theory for very fast voltage sweeps $(1 / \beta \leqslant 50 \mu \mathrm{s} / \mathrm{V})$. These deviations originate from a flattening of the triangular voltage ramps. Records of the triangular voltage pulse taken by an oscilloscope revealed a flattening of the voltage apex depending on the peak voltage $U_{\mathrm{p}}$, which becomes significant for very fast sweeps. This flattening translates into a peak voltage cutoff and hence a different value of $U_{\mathrm{p}}$, which is transduced to the sample. We attribute this to the limited bandwidth of the summation amplifier, which reduces the pulse fidelity for very short ramp times. In the experiments conducted on sample A, a highperformance summation amplifier has been employed together with a different arbitrary function generator. The latter exhibits a greatly enhanced bandwidth and sampling rate (nearly one order of magnitude) compared to the device employed in the room-temperature experiment. As a consequence, the flattening of the voltage pulse apex is less pronounced, and we find good agreement between the experimental data and the theory for inverse voltage sweep rates $1 / \beta \leqslant 50 \mu \mathrm{s} / \mathrm{V}$.
[1] E. C. G. Stückelberg, Theorie der unelastischen Stösse zwischen Atomen, Helv. Phys. Acta 5, 369 (1932).

[2] S. N. Shevchenko, S. Ashhab, and F. Nori, Landau-ZenerStückelberg interferometry, Phys. Rep. 492, 1 (2010).

[3] S. Yoakum, L. Sirko, and P. M. Koch, Stückelberg Oscillations in the Multiphoton Excitation of Helium Rydberg Atoms: Observation with a Pulse of Coherent Field and Suppression by Additive Noise, Phys. Rev. Lett. 69, 1919 (1992).

[4] M. Mark, T. Kraemer, P. Waldburger, J. Herbig, C. Chin, H.-C. Nägerl, and R. Grimm, Stückelberg Interferometry with Ultracold Molecules, Phys. Rev. Lett. 99, 113201 (2007).

[5] E. Dupont-Ferrier, B. Roche, B. Voisin, X. Jehl, R. Wacquez, M. Vinet, M. Sanquer, and S. De Franceschi, Coherent Coupling of two Dopants in a Silicon Nanowire Probed by LandauZener-Stückelberg Interferometry, Phys. Rev. Lett. 110, 136802 (2013).

[6] W. Wernsdorfer, R. Sessoli, A. Caneschi, D. Gatteschi, and A. Cornia, Nonadiabatic Landau-Zener tunneling in $\mathrm{Fe}_{8}$ molecular nanomagnets, Europhys. Lett. 50, 552 (2000).

[7] J. R. Petta, H. Lu, and A. C. Gossard, A coherent beam splitter for electronic spin states, Science 327, 669 (2010).

[8] L. Gaudreau, G. Granger, A. Kam, G. C. Aers, S. A. Studenikin, P. Zawadzki, M. Pioro-Ladriere, Z. R. Wasilewski, and A. S. Sachrajda, Coherent control of three-spin states in a triple quantum dot, Nat. Phys. 8, 54 (2012).
[9] H. Ribeiro, G. Burkard, J. R. Petta, H. Lu, and A. C. Gossard, Coherent Adiabatic Spin Control in the Presence of Charge Noise Using Tailored Pulses, Phys. Rev. Lett. 110, 086804 (2013).

[10] F. Forster, G. Petersen, S. Manus, P. Hänggi, D. Schuh, W. Wegscheider, S. Kohler, and S. Ludwig, Characterization of Qubit Dephasing by Landau-Zener-Stückelberg-Majorana Interferometry, Phys. Rev. Lett. 112, 116803 (2014).

[11] W. D. Oliver, Y. Yu, J. C. Lee, K. K. Berggren, L. S. Levitov, and T. P. Orlando, Mach-Zehnder interferometry in a strongly driven superconducting qubit, Science 310, 1653 (2005).

[12] M. Sillanpää, T. Lehtinen, A. Paila, Y. Makhlin, and P. Hakonen, Continuous-Time Monitoring of Landau-Zener Interference in a Cooper-Pair Box, Phys. Rev. Lett. 96, 187002 (2006).

[13] M. D. Lahaye, J. Suh, P. M. Echternach, K. C. Schwab, and M. L. Roukes, Nanomechanical measurements of a superconducting qubit, Nature (London) 459, 960 (2009).

[14] S. N. Shevchenko, S. Ashhab, and F. Nori, Inverse LandauZener-Stückelberg problem for qubit-resonator systems, Phys. Rev. B 85, 094502 (2012).

[15] M. Gong, Y. Zhou, D. Lan, Y. Fan, J. Pan, H. Yu, J. Chen, G. Sun, Y. Yu, S. Han, and P. Wu, Landau-Zener-Stückelberg-Majorana interference in a 3D transmon driven by a chirped microwave, Appl. Phys. Lett. 108, 112602 (2016). 
[16] G. Heinrich, J. G. E. Harris, and F. Marquardt, Photon shuttle: Landau-Zener-Stückelberg dynamics in an optomechanical system, Phys. Rev. A 81, 011801 (2010).

[17] N. V. Vitanov and B. M. Garraway, Landau-Zener model: Effects of finite coupling duration, Phys. Rev. A 53, 4288 (1996).

[18] L. D. Landau, Zur theorie der energieübertragung. II, Physics of the Soviet Union 2, 46 (1932).

[19] C. Zener, Non-adiabatic crossing of energy levels, Proc. R. Soc. London, Ser. A 137, 696 (1932).

[20] E. Majorana, Atomi orientati in campo magnetico variabile, Nuovo Cimento 9, 43 (1932).

[21] H. Okamoto, A. Gourgout, C.-Y. Chang, K. Onomitsu, I. Mahboob, E. Y. Chang, and H. Yamaguchi, Coherent phonon manipulation in coupled mechanical resonators, Nat. Phys. 9, 480 (2013).

[22] T. Faust, J. Rieger, M. J. Seitner, J. P. Kotthaus, and E. M. Weig, Coherent control of a classical nanomechanical two-level system, Nat. Phys. 9, 485 (2013).

[23] A. B. Shkarin, N. E. Flowers-Jacobs, S. W. Hoch, A. D. Kashkanova, C. Deutsch, J. Reichel, and J. G. E. Harris, Optically Mediated Hybridization Between Two Mechanical Modes, Phys. Rev. Lett. 112, 013602 (2014).

[24] T. Faust, J. Rieger, M. J. Seitner, P. Krenn, J. P. Kotthaus, and E. M. Weig, Nonadiabatic Dynamics of two Strongly Coupled Nanomechanical Resonator Modes, Phys. Rev. Lett. 109, 037205 (2012).

[25] H. J. Maris and Q. Xiong, Adiabatic and nonadiabatic processes in classical and quantum mechanics, Am. J. Phys. 56, 1114 (1988).

[26] B. W. Shore, M. V. Gromovyy, L. P. Yatsenko, and V. I. Romanenko, Simple mechanical analogs of rapid adiabatic passage in atomic physics, Am. J. Phys. 77, 1183 (2009).

[27] G. Sun, X. Wen, B. Mao, Y. Yu, J. Chen, W. Xu, L. Kang, P. Wu, and S. Han, Landau-Zener-Stückelberg interference of microwave-dressed states of a superconducting phase qubit, Phys. Rev. B 83, 180507(R) (2011).

[28] J. Rieger, T. Faust, M. J. Seitner, J. P. Kotthaus, and E. M. Weig, Frequency and $\mathrm{Q}$ factor control of nanomechanical resonators, Appl. Phys. Lett. 101, 103110 (2012).

[29] T. Faust, P. Krenn, S. Manus, J. P. Kotthaus, and E. M. Weig, Microwave cavity-enhanced transduction for plug and play nanomechanics at room temperature, Nat. Commun. 3, 728 (2012).
[30] L. Novotny, Strong coupling, energy splitting, and level crossings: A classical perspective, Am. J. Phys. 78, 1199 (2010).

[31] V.I. Arnold, Mathematical Methods of Classical Mechanics, 2nd ed. (Springer, New York, 1989), Pt. III, Chap. 8, Sec. 38.

[32] M. J. Seitner, H. Ribeiro, J. Kölbl, T. Faust, and E. M. Weig, Finite time Stückelberg interferometry with nanomechanical modes, arXiv:1609.05023 [cond-mat.mes-hall].

[33] T. Usuki, Theoretical study of Landau-Zener tunneling at the M+N level crossing, Phys. Rev. B 56, 13360 (1997).

[34] M. O. Scully and M. Suhail Zubairy, Quantum Optics (Cambridge University Press, Cambridge, 1997).

[35] A. D. O'Connell, M. Hofheinz, M. Ansmann, R. C. Bialczak, M. Lenander, E. Lucero, M. Neeley, D. Sank, H. Wang, M. Weides, J. Wenner, J. M. Martinis, and A. N. Cleland, Quantum ground state and single-phonon control of a mechanical resonator, Nature (London) 464, 697 (2010).

[36] J. D. Teufel, T. Donner, D. Li, J. W. Harlow, M. S. Allman, K. Cicak, A. J. Sirois, J. D. Whittaker, K. W. Lehnert, and R. W. Simmonds, Sideband cooling of micromechanical motion to the quantum ground state, Nature (London) 475, 359 (2011).

[37] H. Xu, D. Mason, L. Jiang, and J. G. E. Harris, Topological energy transfer in an optomechanical system with exceptional points, Nature (London) 537, 80 (2016).

[38] N. Rossi, F. R. Braakman, D. Cadeddu, D. Vasyukov, G. Tütüncüoglu, A. F. i Morral, and M. Poggio, Vectorial scanning force microscopy using a nanowire sensor, Nat. Nanotechnol. (2016), doi:10.1038/nnano.2016.189.

[39] L. Mercier de Lépinay, B. Pigeau, B. Besga, P. Vincent, P. Poncharal, and O. Arcizet, A universal and ultrasensitive vectorial nanomechanical sensor for imaging 2D force fields, Nat. Nanotechnol. (2016), doi:10.1038/nnano.2016.193.

[40] M. R. Andrews, C. G. Townsend, H.-J. Miesner, D. S. Durfee, D. M. Kurn, and W. Ketterle, Observation of interference between two Bose condensates, Science 275, 637 (1997).

[41] Y. Castin and J. Dalibard, Relative phase of two Bose-Einstein condensates, Phys. Rev. A 55, 4330 (1997).

[42] C. Kohstall, S. Riedl, E. R. Sánchez Guajardo, L. A. Sidorenkov, J. H. Denschlag, and R. Grimm, Observation of interference between two molecular Bose-Einstein condensates, New J. Phys. 13, 065027 (2011)

[43] O. Maillet, F. Vavrek, A. D. Fefferman, O. Bourgeois, and E. Collin, Classical decoherence in a nanomechanical resonator, New J. Phys. 18, 073022 (2016). 